\title{
Cryosurgery as adjuvant therapy in cutaneous sporotrichosis
}

Sporotrichosis is a subacute or chronic disease that affects animals and humans caused by the dimorphic fungus Sporothrix schenkii. ${ }^{1}$ An epidemic of sporotrichosis has been noticed in the city of Rio de Janeiro from 1998 onwards and having the cat as the main link in the epidemiological chain. ${ }^{2} \mathrm{We}$ have missed a large proportion of these patients and adjuvant cryosurgery with liquid

Figure 1: Sporotrichosis: pre-treatment.
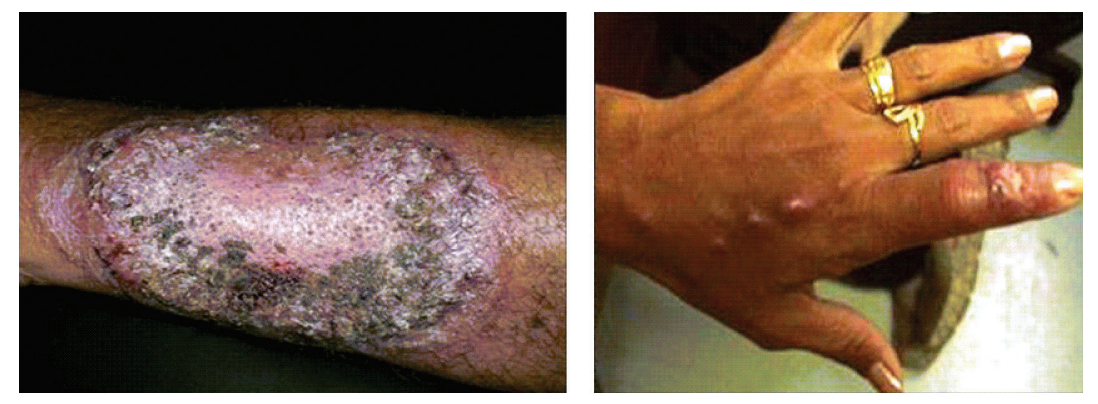

Figure 2: Sporotrichosis: before cryosurgery.

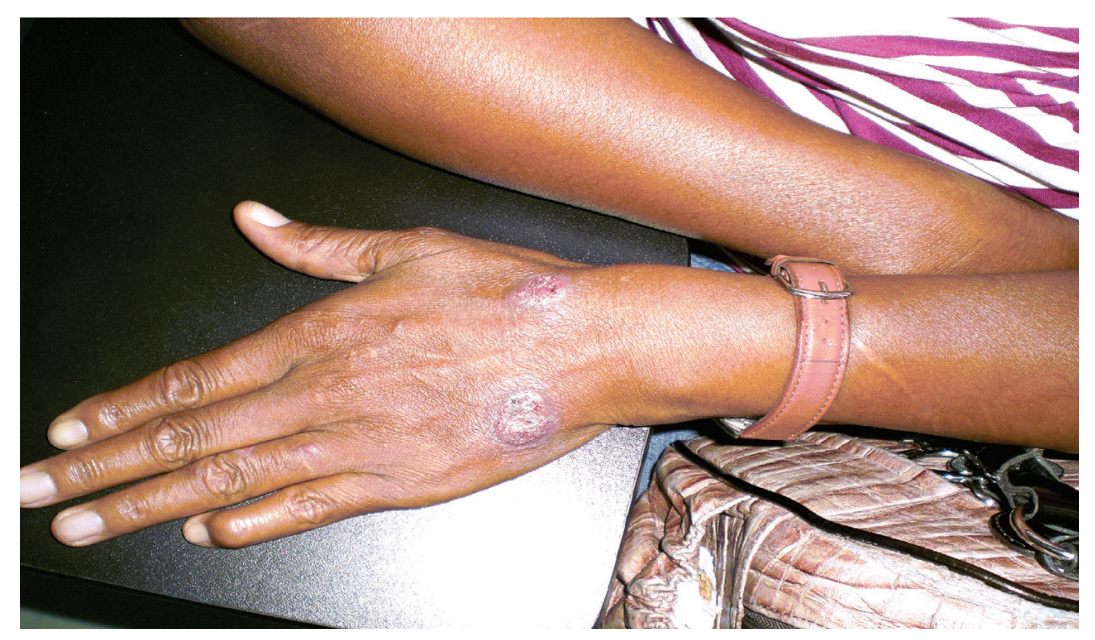

Authors

Cassio Porto Ferreira ${ }^{1}$

Maria Clara Gutierrez

Galhardo ${ }^{2}$

Antônio Carlos

Francescone do Valle

${ }^{1} \mathrm{MSc}$, Techonologist;

Dermatologist,

Laboratory of Clinical Research in Infectious

Dermatology (IPEC/ FIOCRUZ)

${ }^{2}$ Post-Doctorate;

Researcher, Laboratory of Clinical Research in Infectious Dermatology (IPEC/FIOCRUZ)

${ }^{3} \mathrm{PhD}$; Researcher, Laboratory of Clinical Research in Infectious Dermatology (IPEC/ FIOCRUZ)
Submitted on: 9/29/2010 Approved on: 10/5/2010

Correspondence to: Cassio Porto Ferreira Av. Brasil 4365, Manguinhos Rio de Janeiro, Brazil 21040-900

Fax: 00.55-21-2209-4110 Phone:

00.55-21-3865-8102 drcassioferreira@yahoo. com.br 
had isolated Sporothrix schenckii in culture (Figure 3). Results: Patients underwent monthly sessions of cryosurgery with liquid nitrogen with two cycles of 15 seconds and halo of $5 \mathrm{~mm}$ (Figures 4 and 5) with an average of 2.2 sessions (range 1-4 sessions). Five patients had exclusive use of itraconazole for a mean period of 28.8 weeks (range 4-56 weeks) and after the introduction of adjuvant therapy were discharged after a mean period of 12 weeks (range 4-36 weeks). The patient who made use of potassium iodide was discharged after two adjuvant therapy ses- sions, totaling 12 weeks of treatment. The three patients who used itraconazole and terbinafine, itraconazole used primarily by an average of 21.2 weeks and due to lack of response initiated terbinafine, which when combined with adjuvant therapy were discharged after a mean period of 16 weeks (range 4-28 weeks). In conclusion, cryosurgery with liquid nitrogen ${ }^{4}$ used in patients with slower response to systemic antifungal agents in a few sessions reduces treatment time and thus reducing cost and side effects.

[Braz J Infect Dis 2011;15(2):181-183]@Elsevier Editora Ltda.

Figure 3: Dimorphic fungus Sporothrix schenckii. (A): mycelium form (250C); (B): yeast form (370C).

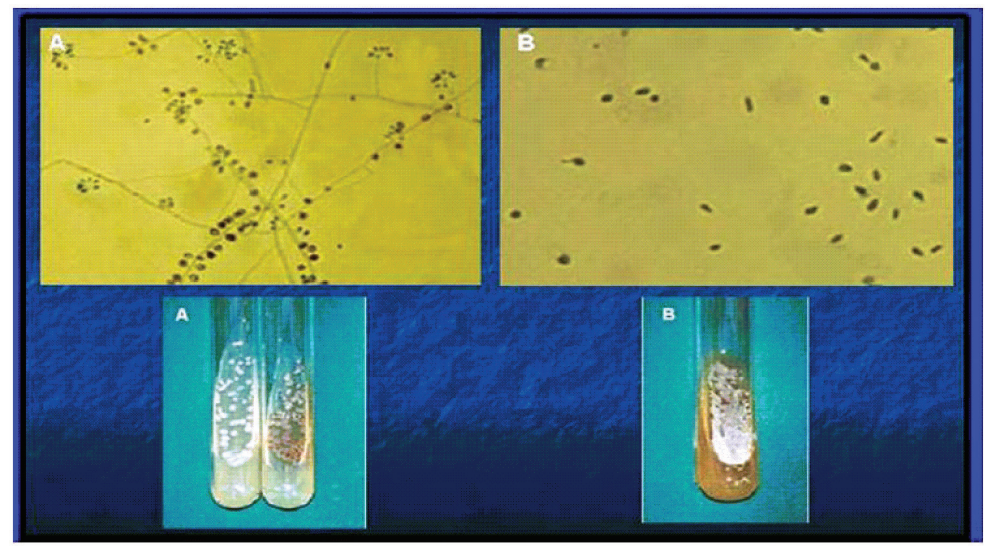

Figure 4: Technique of cryosurgery with liquid nitrogen and halo.

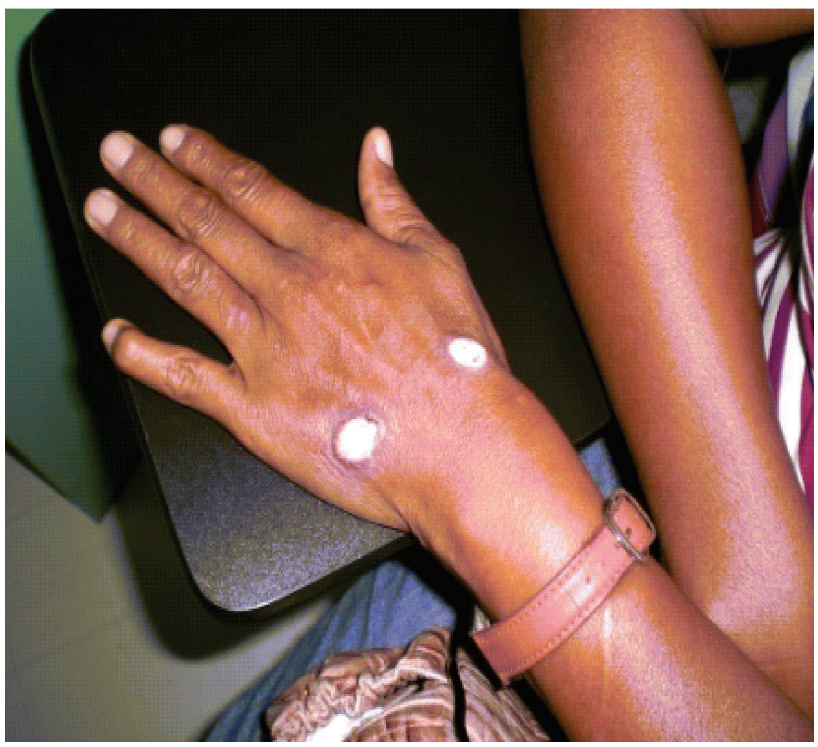

Figure 5: Sporotrichosis: post-treatment.
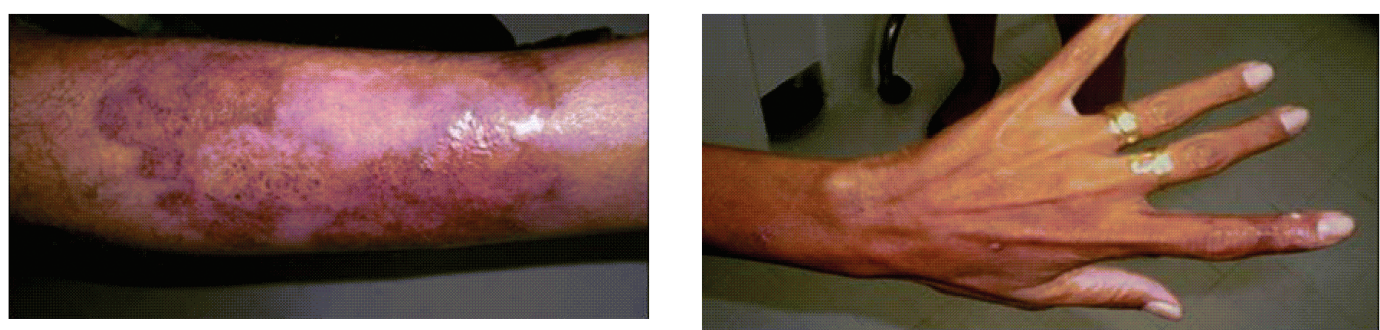


\section{REFERENCES}

1. de Lima Barros MB, de Oliveira Schubach A, Galhardo MC et al. Sporotrichosis with widespread cutaneous lesions: report of 24 cases related to transmission by domestic cats in Rio de Janeiro, Brazil. Int J Dermatol 2003; 42:677-681.

2. Barros MB, Schubach A de O, do Valle AC et al. Cat-transmitted sporotrichosis epidemic in Rio de Janeiro, Brazil: description of a series of cases. Clin Infect Dis. 2004; 38:529-35.
3. Moraes AM, Velho PENFV, Magalhães RF. Criocirurgia com nitrogênio líquido e as dermatoses infecciosas. An Bras Dermatol. 2008; 83(4):285-98.

4. Bargman H. Successful treatment of cutaneous sporotrichosis with liquid nitrogen: report of three cases. Mycoses; 1995; 38:285-7. 